\title{
Evolutionary Meta-Analysis of Solanaceous Resistance Gene and Solanum Resistance Gene Analog Sequences and a Practical Framework for Cross-Species Comparisons
}

\author{
Edmund A. Quirin, ${ }^{1}$ Harpartap Mann, ${ }^{1}$ Rachel S. Meyer, ${ }^{2}$ Alessandra Traini, ${ }^{3}$ Maria Luisa Chiusano, ${ }^{3}$ \\ Amy Litt, ${ }^{2}$ and James M. Bradeen ${ }^{1}$ \\ ${ }^{1}$ University of Minnesota, Department of Plant Pathology, 495 Borlaug Hall/1991 Upper Buford Circle, St. Paul, MN 55108, \\ U.S.A.; ${ }^{2}$ The New York Botanical Garden, Bronx, NY 10458, U.S.A.; ${ }^{3}$ University of Naples Federico II, Dept. of Soil, Plant, \\ Environmental and Animal Production Sciences, Via Università 100, Portici 80055, Italy
}

Submitted 23 December 2011. Accepted 23 January 2012.

Cross-species comparative genomics approaches have been employed to map and clone many important disease resistance $(R)$ genes from Solanum species-especially wild relatives of potato and tomato. These efforts will increase with the recent release of potato genome sequence and the impending release of tomato genome sequence. Most $\boldsymbol{R}$ genes belong to the prominent nucleotide binding site-leucine rich repeat (NBS-LRR) class and conserved NBS-LRR protein motifs enable survey of the $R$ gene space of a plant genome by generation of resistance gene analogs (RGA), polymerase chain reaction fragments derived from $R$ genes. We generated a collection of 97 RGA from the disease-resistant wild potato $S$. bulbocastanum, complementing smaller collections from other Solanum species. To further comparative genomics approaches, we combined all known Solanum RGA and cloned solanaceous NBS-LRR gene sequences, nearly 800 sequences in total, into a single meta-analysis. We defined $R$ gene diversity bins that reflect both evolutionary relationships and DNA cross-hybridization results. The resulting framework is amendable and expandable, providing the research community with a common vocabulary for present and future study of $R$ gene lineages. Through a series of sequence and hybridization experiments, we demonstrate that all tested $\boldsymbol{R}$ gene lineages are of ancient origin, are shared between Solanum species, and can be successfully accessed via comparative genomics approaches.

Potato (Solanum tuberosum L.) and tomato (S. lycopersicum L.) are crop species of worldwide significance, and active breeding communities exist for both. A major goal for breeders is enhanced disease resistance. Potato, for example, is host to more than 60 diseases of contemporary significance (Stevenson et al. 2001). Wild relatives of potato and tomato are rich sources of disease resistance $(R)$ genes, and several have been widely employed by breeders. Supporting plant improvement efforts,

Nucleotide sequence data for Solanum bulbocastanum resistance-gene analogs are available in the GenBank database under accession numbers JQ739520 to JQ739616.

Corresponding author: James M. Bradeen; E-mail: jbradeen@umn.edu

* The $e$-Xtra logo stands for "electronic extra" and indicates that two supplementary figures and two supplementary tables are published online. many Solanum $R$ genes have been mapped and dozens have been cloned (Bradeen 2011; Gebhardt and Valkonen 2001). In these efforts, cross-species comparative genomics-the application of knowledge about genome structure and DNA sequence from one species to further the study of a related species, has played an increasingly prominent role. This is especially true for wild relatives of potato and tomato. The potato genome sequence has been published (Potato Genome Sequencing Consortium 2011), and a draft sequence of tomato is available to the research community. These resources will serve to make comparative genomics approaches to $R$-gene mapping and cloning more accessible to the Solanum research community, speeding the pace of progress (Bradeen 2011). Importantly, however, while previous research has taught us that some $R$ gene lineages are of ancient origin predating speciation events in Solanum - and, thus, are shared between species and can be accessed using comparative genomics approaches-no crossspecies genome-wide survey of the Solanum $R$ gene space has been published to date.

The majority of cloned disease $R$ genes encode both a nucleotide binding site (NBS) and a leucine rich repeat (LRR). Although NBS-LRR genes may vary considerably at a global DNA sequence level, specific motifs encoded in both the NBS and LRR regions are well conserved at a protein level across gene families and plant species. By aligning protein sequences of the tobacco $N$ and the Arabidopsis RPS2 genes, Leister and associates (1996) identified conserved motifs and developed corresponding degenerate and nondegenerate polymerase chain reaction (PCR) primers. Applying these primers to genomic template DNA from potato yielded fragments with homology to known $R$ genes. $R$-gene fragments generated via this approach are called resistance gene analogs (RGA), and the method has been widely applied to both monocot and dicot plant species (Hattendorf and Debener 2007; He et al. 2004; Maleki et al. 2003; Whitaker et al. 2010).

Solanum bulbocastanum Dunal is a diploid $(2 n=2 x=24)$ wild potato species native to central and southern Mexico. Long celebrated for its resistance to the potato and tomato late blight pathogen Phytophthora infestans (Mont.) de Bary-a product of a long co-evolutionary relationship, S. bulbocastanum has been the target of recent $R$-gene mapping and cloning efforts. At least four late blight $R$ genes have been cloned from S. bulbocastanum (Lokossou et al. 2009; Oosumi et al. 2009; Song et al. 2003; van der Vossen et al. 2003, 2005). Each of 
these genes encodes an NBS-LRR protein. The species is also a source of resistance to an array of other important potato diseases, including those caused by viruses, bacteria, fungi, and nematodes (Austin et al. 1993; Brown et al. 1996, 2009; United States Department of Agriculture-Agricultural Research Service [USDA-ARS] National Plant Germplasm System website; unpublished observations).

We have generated an RGA resource for $S$. bulbocastanum, complementing smaller RGA resources previously developed for other Solanum species. Towards facilitating future crossspecies comparative genomics, we developed a curated, practical framework for evolutionary analyses that accurately predicts DNA sequence relationships and DNA cross-hybridization results. $R$-gene lineages tested in sequence and cross-hybridization experiments were shown to be of ancient origin. These lineages, having emerged prior to speciation events, are shared across Solanum species. Significantly, each of the $R$-gene lineages tested retained greater than $80 \%$ NBS DNA sequence identity across species, despite separation by as long as 12 million years. Our research predicts that comparative genomics approaches targeting $R$ genes are generally applicable across a wide array of Solanum species and provides the scientific community with an expandable, amendable, curated resource for current and future analyses.

\section{RESULTS}

\section{Generation and comparison}

of $S$. bulbocastanum RGA sequences.

In this study, we employed four PCR primer pairs targeting conserved motifs in the NBS region of NBS-LRR genes. These included one nondegenerate and seven partially degenerate primers (Table 1). Primers were applied to genomic DNA template and cDNA template from the disease-resistant wild potato S. bulbocastanum genotype PT29, and a stringent definition for classifying amplified fragments as RGA was employed. In total, 97 unique $S$. bulbocastanum RGA were recovered. A majority ( 87 of the $97 ; 89.7 \%$ ) of RGA sequences originated from primer pair SM. In part, these results reflect greater sequencing emphasis on fragments generated using primer pair SM (309 clones) than any other primer pair (85 to 124 clones). However, on a percentage basis, recovery of RGA was also most efficient using primers SM (31.7\% vs. 1.8 to $4.8 \%$ ). Recovery of RGA sequences from $S$. bulbocastanum was complicated by amplification of non-RGA sequences - especially 2D8, a family of highly repetitive elements related to the intergenic spacer region of ribosomal RNA genes (Stupar et al. 2002). Similar complications have been reported for cultivated potato (Leister et al. 1996). In total, 397 cloned fragments originating from genomic DNA and 235 cloned fragments originating from cDNA were sequenced. Of 97 unique sequences classified as RGA, 82 $(84.5 \%)$ originated from genomic DNA template and 15 $(15.5 \%)$ originated from cDNA template. Within a plant genome, many NBS-LRR loci exist as truncated gene copies or may encode premature stop codons or frameshift mutations that will eliminate proper $\mathrm{R}$ protein function. RGA originating from cDNA template represent transcribed (not necessarily translated) genes, while RGA originating from genomic DNA may or may not be transcribed. Since $S$. bulbocastanum is a diploid, obligate outcrossing and highly heterozygous species, distinct RGA fragments represent distinct $R$-gene alleles but may or may not originate from distinct $R$-gene loci. The 97 RGA sequences reported here for $S$. bulbocastanum compose the largest RGA resource reported for any Solanum species to date.

To explore phylogenetic relationships between $S$. bulbocastanum RGA, neighbor-joining and parsimony analyses of translated protein sequences were employed. Parsimony analysis assumes equal rates of evolution across all RGA fragments, while neighbor-joining analysis does not. Because $R$-gene families may evolve at vastly different rates, neighbor-joining has been more widely employed for $R$-gene evolutionary analyses (Cannon et al. 2002; Meyers et al. 2003; Zhou et al. 2004). Nevertheless, results generated by these two approaches were largely concordant (not shown). Results of our neighborjoining analysis are shown in Figure 1.

One prominent feature of our analysis is a broad, well-supported distinction between RGA putatively originating from NBS-LRR genes that also encode a Toll/interleukin-like receptor (TIR) and those that do not (non-TIR). While TIR and nonTIR proteins differ prominently at the $\mathrm{N}$-terminus, specific motifs in the NBS region are also associated with these gene subclasses. TIR- and non-TIR NBS-LRR lineages are thought to have ancient evolutionary origins (Meyers et al. 2002). The genomes of all dicots surveyed to date encode both TIR- and non-TIR NBS-LRR proteins (Meyers et al. 2003), but the genomes of grasses contain only non-TIR NBS-LRR genes (Zhou et al. 2004). Consistent with previous findings (Cannon et al. 2002), our analysis reveals deep evolutionary divisions within the non-TIR genes. We recovered 89 TIR-NBS-LRR and eight non-TIR NBS-LRR RGA from $S$. bulbocastanum. The preponderance of TIR-NBS-LRR genes in our study likely reflects, in part, bias in our technical approach rather than a biological preference for TIR-type genes. In particular, among the four primer pairs surveyed in this study, primer pair SM targets motifs associated with TIR-NBS-LRR genes while the other primer pairs target motifs found in non-TIR NBS-LRR genes. Because a large majority $(89.7 \%)$ of the RGA sequences generated in this study were amplified by primer pair SM, our data set is skewed towards TIR-NBS-LRR genes. While Meyers and associates (2003) noted an excess of TIR-NBS-LRR genes in the Arabidopsis genome, analysis of complete genome sequence of cultivated potato-a closer relative of $S$. bulbocastanum-reveals an excess of non-TIR NBS-LRR genes (Potato Genome Sequencing Consortium 2011). A more thorough survey of the non-TIR NBS-LRR gene space of $S$. bulbocastanum is warranted.

With the goal of organizing S. bulbocastanum RGA sequences into groupings that reflect evolutionary relationships

Table 1. Primer sequences used for polymerase chain reaction amplification of Solanum bulbocastanum resistance gene analogs

\begin{tabular}{|c|c|c|c|c|}
\hline Pair code & Primer name & 5'-Primer sequence- $3^{\prime a}$ & Motif $^{\text {b }}$ & Reference \\
\hline \multirow[t]{2}{*}{$\mathrm{bb}$} & bliC1 & CGT GGG CAT CTG GGG NAT GNS NGG & VSIWGMPG & This study \\
\hline & bliF1 & TCG GTG TGC CAG ACG TCR TCN ADN AC & VLDDVWHTD & \\
\hline \multirow[t]{2}{*}{ PP } & ModP-F & GGI AAR ACI ACI YTI GCI CAR ATG & GKTTLAQM & L. Boiteux, unpublished \\
\hline & ModP-R & GRT AIS WIA RIC KIA RIG CIG G & PALRLSY & \\
\hline \multirow[t]{2}{*}{ SA } & s1 & GGT GGG GTT GGG AAG ACA ACG & GGVGKTT & Leister et al. 1996 \\
\hline & as $2 \mathrm{I}$ & IAA IGC IAG IGG IAA ICC & GLPLAL & \\
\hline \multirow[t]{2}{*}{ SM } & $\mathrm{s} 2 \mathrm{~N}$ & GGN GGN GTN GGN AAN CAN AC & GGVGKTT & Leister et al. 1996 \\
\hline & Motif1 & GAA GCA IGC GAT GTC IAG GAA & FLDIACF & Lopez et al. 2003 \\
\hline
\end{tabular}

\footnotetext{
${ }^{\mathrm{a}}$ Codes for degenerate positions: $\mathrm{I}=$ inosine; $\mathrm{N}=\mathrm{A}, \mathrm{C}, \mathrm{G}$ or $\mathrm{T} ; \mathrm{R}=\mathrm{A}$ or $\mathrm{G} ; \mathrm{Y}=\mathrm{C}$ or $\mathrm{T} ; \mathrm{W}=\mathrm{A}$ or $\mathrm{T} ; \mathrm{S}=\mathrm{G}$ or $\mathrm{C} ; \mathrm{D}=\mathrm{A}, \mathrm{G}$, or $\mathrm{T}$; $\mathrm{K}=\mathrm{G}$ or $\mathrm{T}$.

${ }^{b}$ Amino acid sequence of NBS motifs used for primer design. The most highly conserved portions are shown in bold.
} 
and origins, we assigned DNA sequences to contigs at an $80 \%$ sequence identity cutoff. Using this approach, eight contigs composed of two or more sequences and six singletons were identified. We refer to these groupings as "Sblb80" (for " $S$. bulbocastanum at $80 \%$ sequence identity") diversity bins and assigned sequential numbers to each group (Fig. 1). Importantly, superimposition of Sblb80 contig information onto the

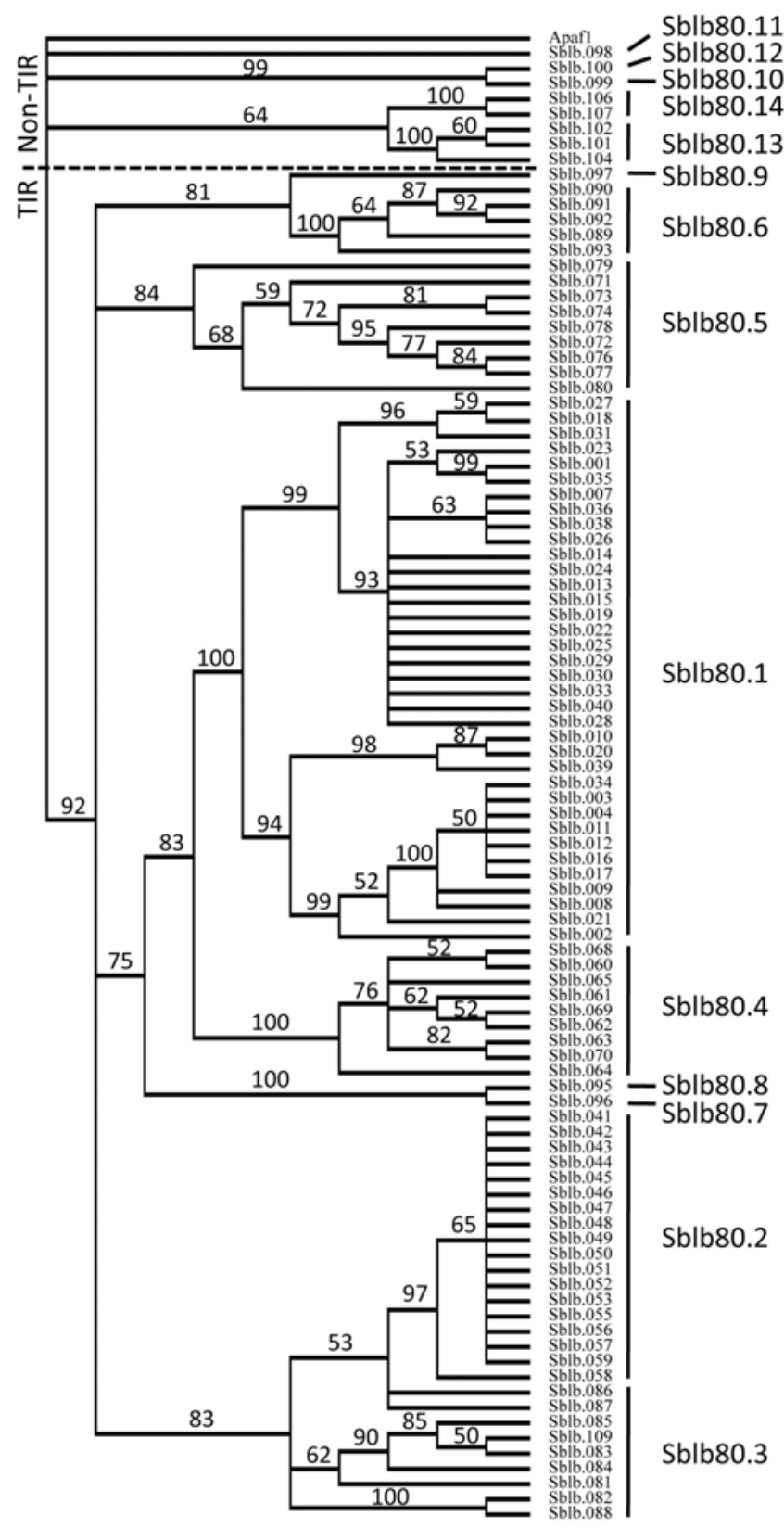

Fig. 1. A neighbor-joining tree of Solanum bulbocastanum resistance gene analog (RGA) protein sequences. DNA sequences from 97 S. bulbocastanum RGA clones were translated to protein sequences and were aligned using MUSCLE V. 3.8. The protein sequence of human Apoptotic protease activating factor 1 (Apaf1) was used as an outgroup. Numbers above branches indicate bootstrap percentages based on 1,000 replications Neighbor-joining analysis reveals broad separation of Toll/interleukin-like receptor (TIR) and non-TIR RGA, with TIR RGA being especially well represented. DNA sequences were assigned to Sblb80 diversity bins $(80 \%$ DNA identity contigs) as indicated. Without exception, Sblb80 bins defined on the basis of DNA identities correspond to clades in the neighborjoining analysis of protein sequences. Sblb80 bins differ in size from a single sequence to 36 sequences. This is likely due both to variation in allele copy number across lineages and to variation in primer efficiency across lineages. neighbor-joining tree revealed that, without exception, each Sblb80 bin forms a monophyletic clade (Fig. 1). Frequently, these Sblb80 clades are distinguished with strong bootstrap support. Thus, applying an $80 \%$ DNA sequence identity cutoff to define RGA families reflects inferred evolutionary relationships of protein sequences.

Our approach resulted in diversity bins ranging in size from a single sequence (a singleton) to one bin (Sblb80.1) comprising 36 sequences. Differences in Sblb80 diversity bin sizes may reflect both differences in the physical size of gene clusters within the genome of $S$. bulbocastanum and differences in the efficiency of PCR amplification across NBS-LRR gene lineages. Since $S$. bulbocastanum is a diploid, the maximum number of alleles at any $R$-gene locus is two. Thus, the number of $R$-gene loci sampled for each Sblb80 group is at least one half the number of recovered RGA sequences. For Sblb80.1, therefore, we have surveyed alleles at a minimum of $18(36 / 2)$ distinct $R$-gene loci. We speculate that high allelic diversification of the SolaR80.1 lineage in $S$. bulbocastanum provides the opportunity for functional diversification of this gene lineage. The ratio of nonsynonymous $(\mathrm{Ka})$ to synonymous $(\mathrm{Ks})$ nucleotide substitutions is an indicator of positive selection for protein modifications yielding new functions (Liberles 2001; Siltberg and Liberles 2002). We calculated $\mathrm{Ka} / \mathrm{Ks}$ ratios for $S$. bulbocastanum SolaR80.1 RGA sequences. For most pairs of RGA sequences, observed $\mathrm{Ka} / \mathrm{Ks}$ ratios were statistically indistinguishable from 0 , consistent with selective pressure retaining both protein sequence and function. However, comparison of RGA sequences Sblb.007 and Sblb.028 resulted in a Ka/Ks ratio significantly greater than 1 , suggesting positive diversifying selection. Our results, therefore, are consistent with functional diversification of the SolaR80.1 $R$-gene lineage in $S$. bulbocastanum, a hypothesis that will be explored further in future research.

While our analyses focused on DNA and protein sequences, $R$-gene and RGA research also frequently employs DNA crosshybridization techniques. We empirically tested the level of sequence identity required for successful DNA cross-hybridization by arraying each of our 97 S. bulbocastanum RGA frag-

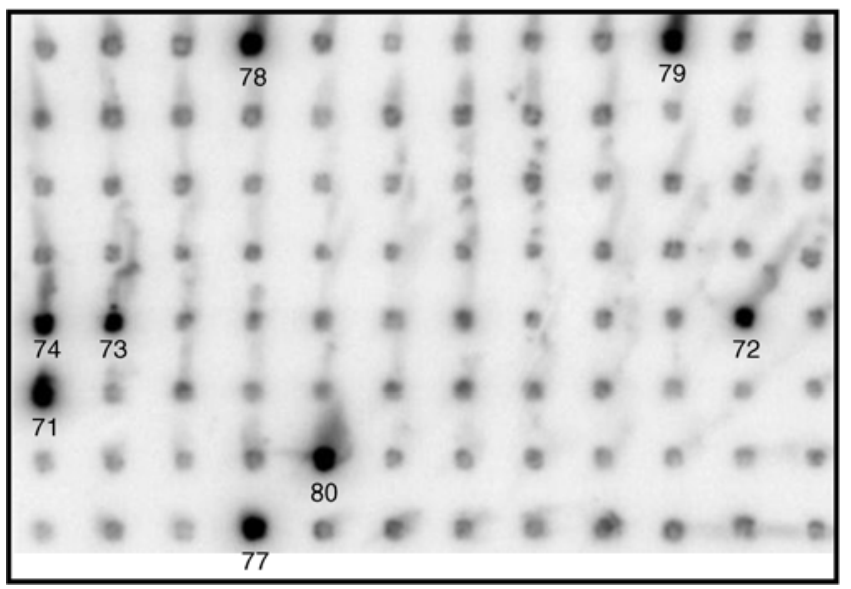

Fig. 2. Cross-hybridization of DNA requires approximately $80 \%$ identity. This autoradiogram shows hybridization of labeled probe Solanum bulbocastanum resistance-gene analog (RGA) Sblb.80 to nylon membrane containing spotted DNA from each $S$. bulbocastanum RGA clone. Clones hybridizing to probe Sblb.80 (dark spots) are indicated by RGA number; each of these clones was assigned on the basis of DNA sequence identity to diversity bin Sblb80.5. All other clones failed to hybridize to probe Sblb.80 and were assigned on the basis of DNA sequence identity to other Sblb80 bins. Results shown are representative of all hybridizations. Thus, DNA cross-hybridization requires approximately 80\% DNA sequence identity, consistent with usage of this threshold for defining RGA lineages. 
ments onto nylon membranes and then probing with representatives from each Sblb80 bin (Fig. 2). Each probe hybridized only to fragments assigned to the same Sblb80 diversity bin; cross-hybridization between bins was not observed nor did any member of a bin fail to hybridize to a probe from the same bin. These results indicate that successful DNA cross-hybridization requires approximately $80 \%$ sequence identity. DNA cross-hybridization results are thus concordant with assigning sequences to diversity bins based on $80 \%$ sequence identity.

Assignment of RGA sequences to diversity bins based on $80 \%$ DNA sequence identity provides a tractable, augmentable, and amendable framework for the study of NBS-LRR gene lineages.

\section{Comparison of RGA and $\boldsymbol{R}$ genes across Solanum species.}

The $S$. bulbocastanum RGA library of 97 unique clones generated in this study complements RGA collections generated for other Solanum species: cultivated tomato ( 73 clones [Pan et al. 2000]), cultivated potato (11 clones [Leister et al. 1996]), cultivated eggplant (S. melongena, nine clones [T. Kiran Babu, K. Raghuprakash, S. Sivaramakrishnan, K. Varaprasad, G. Anuradha, and P. Jayamma, unpublished]), and wild relatives of eggplant ( $S$. aculeatissimum, 11 clones [Zhuang and Wang 2009]), tomato (S. habrochaites, eight clones [Nino-Liu et al. 2003]), potato $(S$. phureja $\times S$. stenotomum, four clones [I. Simko and R.W. Jones, unpublished] and S. demissum, 29 clones [J. Zhou, Y. Zhang, X. Pei, Y. Song, Z. Wang, and S. Jia, unpublished]), and pepino ( $S$. caripense, 40 clones [Trognitz and Trognitz 2005]). While Zhang and associates (2002) report the Southern-based mapping of $29 R$-gene NBS-LRR fragments in tomato, DNA sequences were not generated in that study. Similarly, although Brugmans and associates (2008) reported the generation of 34 RGA from potato via NBS profiling, sequences have not been made publicly available and were not included in our analyses. Finally, Wang and associates (2008) employed NBS profiling to study phylogenetic relationships among 49 tuber-bearing Solanum species. This study documents the broad utility of RGA approaches to study Solanum species and to determine species relationships, but no publicly available DNA sequences were reported from that study.

In addition to the RGA resources described above, more than a dozen functional $R$ genes have been cloned from solanaceous species, especially from Solanum species. Combining sequence data from the NBS region of 472 cloned $R$ genes and $R$-gene homologs and 282 RGA sequences from Solanum species in a single meta-analysis, we defined $80 \%$ DNA sequence identity contigs. In total, we identified 12 contigs composed of one sequence (i.e., a singleton) and 44 contigs composed of two or more sequences. These 56 contigs are referred to as "SolaR80" (for Solanum $R$ gene and $R$ gene-related NBS contigs at $80 \%$ sequence identity) diversity bins and have been sequentially numbered (Fig. 3). We propose community-wide adoption of the SolaR80 terminology and expansion and refinement of our SolaR80 lineages to facilitate future cross-species comparisons.

As was true for S. bulbocastanum RGA (Fig. 1), neighborjoining (Fig. 3) and parsimony (not shown) analyses of consensus protein sequences from each SolaR80 lineage revealed well-supported distinction of non-TIR and TIR SolaR80 lineages. Also evident are deep evolutionary divisions within the non-TIR genes. To confirm that SolaR80 diversity bins reflect inferred evolutionary relationships between corresponding protein sequences, we generated a neighbor-joining tree from a subset of 279 individual $R$-gene and Solanum RGA sequences from 50 different SolaR80 bins (Supplementary Fig. S1). Comparison of this tree with the SolaR80 consensus tree (Fig. 3) reveals broad concordance. In a vast majority of cases, $80 \%$ DNA sequence identity contigs (Fig. 3, SolaR80 consensus sequences) form monophyletic lineages in dendrograms constructed from individual protein sequences. There are, however, two exceptions. SolaR80.37 and SolaR80.53 are each represented by two sequences. In both instances, member sequences associate more closely with sequences from other SolaR80 bins than with sequences from their own SolaR80 bin. SolaR80.37 member AF404469 is loosely (bootstrap value of $58 \%$ ) associated with sequences from SolaR80.35 while SolaR80.37 member AJ009720 is loosely (bootstrap value of $51 \%$ ) associated with SolaR80.6. Similarly, Sblb.97, a member of SolaR80.53, aligns more closely with SolaR80.35 sequences, while AJ716177, also assigned to SolaR80.53, is embedded with SolaR80.37. In both of these examples, the SolaR80 diversity bins involved are closely related. Thus, researchers studying particular $R$-gene or RGA sequences are advised to also consider SolaR80 diversity bins closely related to their target sequences. We anticipate that future incorporation of additional $R$-gene or RGA sequences will lead to refinement of the SolaR80 lineages defined in this study. While it is likely that any such refinements will be minor, they may lead to resolution of the conflicts discussed above.

The recently published genome sequence of a doubled monoploid cultivated potato genotype encompasses 408 NBSLRR genes (Potato Genome Sequencing Consortium 2011). Unfortunately, a complete NBS-LRR gene list has not been made publicly available. Based on reported gene annotations, we identified 394 candidate NBS genes; motif searches confirmed the NBS domain for 206 genes. NBS sequences were manually extracted for each gene. Of the resulting 206 DNA sequences, $88(42.7 \%)$ could be assigned to an existing SolaR80 bin. Contributions per bin varied from one sequence to 20 sequences and, in total, potato genome sequences were assigned to 18 of the 56 defined SolaR80 bins. The remaining 118 NBS sequences were assigned to an additional 53 bins. Most (38 of 53, $71.7 \%$ ) of these bins comprised a single sequence, and the largest bin comprised 20 sequences. We speculate that the majority of NBS sequences from the potato genome that could not be assigned to existing SolaR80 bins represents incremental variants of known Solanum sequences rather than unique, undiscovered $R$-gene lineages. Eventual public release of a complete set of curated NBS sequences from the potato genome will allow incorporation of all potato NBS sequences into the definition of the SolaR80 bins and testing of this hypothesis-efforts we intend to pursue. This effort illustrates the utility and flexibility of our diversity binning approach and documents the need for on-going curation and amendment of the SolaR80 framework.

\section{Conservation of SolaR80 lineages across Solanum species.}

To explore the representation of SolaR80 diversity bins across Solanum species, we superimposed RGA sequence distribution information onto our neighbor-joining analysis of consensus protein sequences (Fig. 3). Of 56 defined SolaR80 bins, $25(44.6 \%)$ are composed solely of RGA generated from one Solanum species and four $(7.1 \%)$ are composed solely of cloned $R$ genes from one Solanum species. Other SolaR80 bins are represented by a combination of RGA or cloned $R$ genes or both from multiple Solanum species. Of particular note, SolaR80.24 is represented by cloned $R$ genes or $R$-gene homologs from 10 different species and RGA recovered from five of the nine Solanum species represented in our study. None of the SolaR80 bins is represented in all nine Solanum species. The species represented in our study reflect a broad sample of the genus and phylogenetic distances between species vary considerably (Fig. 3). Lack of representation of SolaR80 bins 


\section{Resistance Gene Analogs}

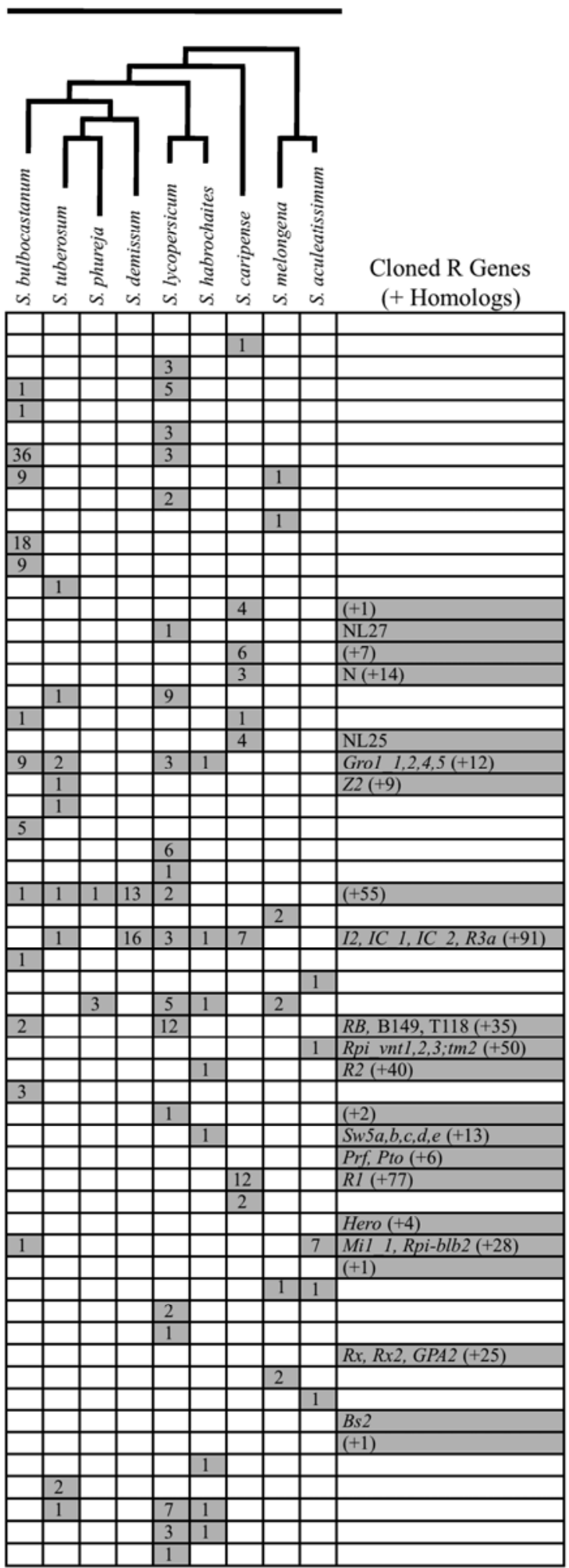

Fig. 3. Neighbor-joining tree of Solanaceous $R$ gene and Solanum resistance gene analogs (RGA) NBS protein sequences. DNA sequences of the NBS region of 472 cloned Solanaceous $R$ genes and $R$ gene homologs and of 282 Solanum RGA were assigned to SolaR80 (80\% DNA identity contigs) diversity bins. Consensus DNA sequence of each SolaR80 bin was translated and the resulting protein sequences were aligned using MUSCLE V. 3.8. Protein sequence of human Apoptotic protease activating factor 1 (Apaf1) was used as an outgroup. Numbers above branches indicate bootstrap percentages based on 1,000 replications. Neighbor-joining analysis reveals broad separation of TIR and non-TIR sequences. The distribution of sequence representation across Solanum species is indicated as a table on the right; shadowed boxes indicate that one or more (as specified) sequences from a given species were assigned to a SolaR80 diversity bin. Inferred Solanum species relationships (Spooner et al. 1993; Weese and Bohs 2007) are indicated by the dendrogram at the top of the figure. Note that RGA fragments generated by I. Simko and R. W. Jones (unpublished) originated from the cross S. phureja X S. stenotomum. In this figure, this cross is indicated as "S. phureja" but species origin for reported RGA is undetermined. Most SolaR80 diversity bins are represented as sequences from a single species, indicating incomplete sampling of the NBS-LRR gene space and/or origination, loss, or divergence of $R$ gene lineages after speciation events. 
across all Solanum species may have several causes. First, RGA sampling has been more aggressive in some species than in others, and it is likely that no Solanum species has been exhaustively sampled to date. The same is true for $R$-gene cloning efforts. Thus, patterns of species representation across SolaR80 diversity bins are likely biased and incomplete. Second, NBS-LRR genes commonly evolve through a process of birth and death (Cannon et al. 2002; Couch et al. 2006; Michelmore and Meyers 1998). It is, therefore, possible that some SolaR80 bins (e.g., SolaR80.24) transcend Solanum speciation, while other lineages are of more recent (i.e., postspeciation) origin. Conversely, specific SolaR80 lineages may have been lost in particular Solanum species postspeciation. Finally, $R$-gene lineages are subject to evolutionary selective pressures imposed by shifting pathogen populations. SolaR80 groupings are based on an $80 \%$ DNA sequence identity threshold. It is possible that $R$ genes that share an evolutionary origin but are represented as RGA from different Solanum species have undergone adaptation, diverging to such a degree that homology falls below $80 \%$.

Knowledge of the distribution of $R$-gene lineages across Solanum species has important implications for crop improvement and comparative genomics approaches. To more thoroughly explore $R$-gene lineage distribution, we performed targeted DNA cross-hybridization experiments. First, on the basis of neighbor-joining analyses, five SolaR80 diversity bins were selected for use as probes to hybridize to Solanum bacterial artificial chromosome (BAC) libraries. Three of these lineages (SolaR80.4, SolaR80.9, and SolaR80.41) are comprised solely of $S$. bulbocastanum RGA. SolaR80.1 includes RGA generated from both $S$. bulbocastanum and cultivated tomato and SolaR80.5 includes RGA from S. bulbocastanum and S. melongena (Fig. 3). Probes consisting of inserts from each constituent $S$. bulbocastanum RGA clone were hybridized to BAC libraries of $S$. bulbocastanum, S. demissum (a wild species closely related to cultivated potato), cultivated tomato, and cultivated eggplant. Table 2 summarizes our hybridization results. Despite spotty representation in RGA libraries, each SolaR80 lineage was represented in the genomes of $S$. bulbocastanum, $S$. demissum, and tomato. While SolaR80.5 and SolaR80.41 hybridized to eggplant BAC clones, SolaR80.1, SolaR80.4, and SolaR80.9 did not. However, hybridization of these three probe sets to genomic Southern blots of eggplant cultivars and related species revealed, despite the BAC-based results, that each of these three $R$-gene lineages is indeed present in the eggplant genome (Supplementary Fig. S2).

On the basis of our various analyses, we conclude that most $R$-gene lineages are present in the genomes of most Solanum species. In other words, $R$-gene lineages, in general, are of ancient origin and, despite allelic diversification, NBS regions still share commonalities detectable using sequenceand hybridization-based approaches. Furthermore, RGA sampling to date in the genus Solanum has been both biased and incomplete.

Table 2. Solanum spp. BAC clones per genome equivalent hybridizing to selected SolaR80 diversity bins

\begin{tabular}{lcccc}
\hline & \multicolumn{4}{c}{ Solanum spp. } \\
\cline { 2 - 5 } SolaR80 bin & bulbocastanum & demissum & lycopersicum & melongena \\
\hline SolaR80.1 & 12.5 & 5.1 & 6.5 & 0.0 \\
SolaR80.4 & 14.2 & 7.6 & 0.9 & 0.0 \\
SolaR80.5 & 7.5 & 2.5 & 4.7 & 1.2 \\
SolaR80.9 & 7.5 & 1.3 & 3.7 & 0.0 \\
SolaR80.41 & 8.3 & 12.7 & 9.8 & 6.0 \\
\hline
\end{tabular}

${ }^{a}$ Average number of BAC clones per genome equivalent observed.

\section{DISCUSSION}

Bonierbale and associates (1988) were the first to note structural similarities between the genomes of potato and tomato, leading to the conclusion that speciation in the genus Solanum correlates with major genome rearrangement events, including chromosome inversions and translocations. Similar observations of genome-wide conservation of marker order have also been extended to other solanaceous species (Doganlar et al. 2002; Livingstone et al. 1999; Prince et al. 1993). By aligning the genomes of potato, tomato, and pepper (genus Capsicum, family Solanaceae) and by superimposing mapping information for phenotypic resistance collected from multiple studies, Grube and associates (2000) demonstrated that common genome regions across these three species condition disease resistance more often than expected by chance. Thus, genome regions conditioning disease resistance have maintained a resistance function throughout speciation. Interestingly, these authors also noted that common regions of the genomes of potato, tomato, and pepper may condition resistance to different pathogen classes (e.g., a virus, a bacterium, and a fungus). Later, Mazourek and associates (2009) showed that orthologs of the potato $R x / G p a 2$ gene cluster map to corresponding genome locations in both tomato and pepper. Thus, $R$ genes of common evolutionary origin and sequence similarity occupy corresponding genome locations throughout solanaceous species. This observation suggests that cross-species comparative genomics approaches to $R$ gene mapping and cloning will be successful in the family. Indeed, comparative genomics approaches have already been widely employed for the cloning of $R$ genes conditioning resistance to Phytophthora infestans (Bradeen 2011). With the release of the complete genome sequence for potato (Potato Genome Sequencing Consortium 2011) and the impending release of the complete genome sequence for tomato, comparative genomics strategies for the mapping and cloning of Solanum $R$ genes are likely to gain traction in coming years.

While previous research demonstrated that specific $R$ genes originated prior to speciation events in the Solanaceae, are shared across species, and can be studied using comparative genomics approaches, our research is the first attempt to explore the cross-species distribution of the entire Solanum $R$ gene space. Using RGA generated from nine Solanum species and $R$ genes cloned from multiple solanaceous species, we have developed the SolaR80 framework. SolaR80 diversity bins are defined on the basis of DNA sequence identity with an $80 \%$ threshold. We have demonstrated that this threshold yields groupings that reflect both protein-based dendrogram topologies and DNA cross-hybridization results. The SolaR80 framework can be easily expanded to encompass additional sequences and can be refined and amended by the broader research community.

In total, we defined 56 SolaR80 diversity bins and demonstrated through sequence and DNA hybridization experiments that each bin is represented in the genomes of several Solanum species. Of particular note, all tested SolaR80 bins were present in the genome of eggplant. Eggplant is the most phylogenetically distinct species represented in our study and is postulated to have diverged from the potato/tomato clade no more than 12 million years ago (Doganlar et al. 2002; Wikstrom et al. 2001). That each of the tested $R$-gene lineages is present in eggplant as well as closer relatives of tomato and potato indicates that i) the $R$-gene lineages originated prior to speciation events in Solanum that distinguished eggplant from potato and tomato (and potentially much earlier), ii) that $R$-gene lineages have been maintained in various Solanum species even after speciation events - an observation that implies retained resistance function, and iii) that diversification of the NBS region 
of these $R$ genes has been slow, allowing detection of at least $80 \%$ sequence similarity. Although our survey of Solanum species is in no way exhaustive, we have selected species differing in their degree of phylogenetic relatedness that represent a broad sample of the genus. Based on our results, we speculate that most $R$-gene lineages will be represented in the genomes of most Solanum species. Thus, our results predict that comparative genomics approaches to $R$-gene mapping and cloning will be generally useful across both $R$-gene lineages and $S o$ lanum species. These findings have significance, especially for agricultural improvement of potato and tomato, since wild Solanum species have been important sources for disease resistance for these crops.

However, our research also suggests that $R$-gene lineages may have undergone major expansion and allelic diversification in specific Solanum species, allowing speculation on the relative significance of $R$-gene lineages to the health of a species. In this study, we targeted the NBS region of NBSLRR genes for cross-species comparisons. The NBS region of the mature NBS-LRR protein is involved primarily in ATP (adenosine triphosphate) hydrolysis and activation of defense signaling (Tameling et al. 2002). Due to this functional constraint, the NBS region is slow to evolve, consistent with our results. In contrast, the LRR region of NBS-LRR proteins is the major determinant of pathogen specificity and even minor changes in LRR sequence can radically alter pathogen specificity (Ellis et al. 1999). To keep pace with ever-changing pathogen populations, the LRR region has a much higher rate of evolution than the NBS region. However, while pathogen specificity is primarily determined by the LRR, intramolecular interactions within the mature $\mathrm{R}$ protein are critical to proper function (Moffett et al. 2002). Thus, changes in the LRR region may also result in coordinated changes in NBS sequence (Couch et al. 2006). Conversely, changes in NBS sequence may be indicative of changes in the associated LRR and, thus, changes in pathogen specificity. In this study, SolaR80.1 shows high allelic diversity in $S$. bulbocastanum genotype PT29, with 36 unique NBS RGA organized into three sublineages (Fig. 3). Interestingly, hybridization of the SolaR80.1 S. bulbocastanum RGA to BAC blots of S. bulbocastanum, $S$. demissum, tomato, and eggplant suggests significant variation in the physical size of gene clusters (and possibly gene copy number) for this lineage in different species (Table 2). In particular, the SolaR80.1 lineage is best represented in $S$. bulbocastanum and representation of SolaR80.1, as indicated by the number of BAC clones per genome equivalent detected, decreases with increasing phylogenetic distance from $S$. bulbocastanum (Table 2). However, because $R$-gene clusters may vary in size even between genotypes of a single species, our results must be interpreted with caution, and a more thorough survey of allelic diversification of the SolaR80.1 lineage in additional genotypes of each species is warranted. With this caveat, we hypothesize that allelic diversification of the SolaR80.1 lineage in S. bulbocastanum genotype PT29 (and possible in $S$. bulbocastanum per se) has been accompanied and probably facilitated by expansion of gene copy numbers-a hypothesis that can be tested when more Solanum complete genome sequences are available and through targeted deep sequencing of the SolaR80.1 lineage in multiple Solanum species. Furthermore, we hypothesize that allelic diversification of the SolaR80.1 lineage in S. bulbocastanum may be indicative of functional significance of this $R$-gene lineage to the health of the species. Consistent with this hypothesis, comparison of most $S$. bulbocastanum Sola$\mathrm{R} 80.1$ sequences yielded $\mathrm{Ka} / \mathrm{Ks}$ ratios close to 0 -indicating selective pressure that maintains protein sequence and associated functionality. In contrast, SbRGA7 and SbRGA28 are associated with a $\mathrm{Ka} / \mathrm{Ks}$ ratio significantly greater than $1-$ an indication of positive diversifying selection. Thus, it appears different members of the SolaR80.1 lineage in $S$. bulbocastanum may play roles in defense against different races or classes of pathogens. To date, no phenotypic function has been reported for any member of the SolaR80.1 lineage.

Our research also reveals, not surprisingly, that RGA methods have not been applied exhaustively to any Solanum species. Analysis of complete genome sequence of the cultivated potato monoploid DM1-3516R44 revealed 408 NBS-LRR genes (Potato Genome Sequencing Consortium 2011). Assuming a similar number of NBS-LRR loci in $S$. bulbocastanum, our RGA collection of 97 unique sequences-the largest collection for any Solanum species, represents no more than $23.7 \%$ of the NBS-LRR genes in the genome. (Due to a high degree of heterozygosity in $S$. bulbocastanum, a diploid, and our inability to differentiate between $R$-gene paralogs vs. alleles derived from a single locus, the degree to which our RGA collection represents all $S$. bulbocastanum NBS-LRR genes might be as low as $11.9 \%$.) Thus, current RGA approaches are under-representing the NBS-LRR gene space of Solanum genomes. The use of next generation sequencing in conjunction with RGA approaches is well poised to facilitate more thorough sampling of the NBSLRR gene space of Solanum species. Interestingly, our S. bulbocastanum RGA collection encompasses 89 TIR-NBS-LRR alleles representing between 48 to 89 distinct loci. In contrast, analysis of genome sequence of the cultivated potato genome revealed 57 TIR-NBS-LRR loci (Potato Genome Sequencing Consortium 2011). Either cultivated potato and S. bulbocastanum differ in the frequency of TIR-NBS-LRR loci harbored or our RGA collection has captured a majority of the TIR-NBSLRR sequences found in the $S$. bulbocastanum genome. These possibilities will be explored when the complete genome sequence is available for $S$. bulbocastanum.

The availability of a high-quality complete genome sequence of both cultivated potato and tomato in the near future will allow anchoring of SolaR80 lineages to specific genome locations and comparison of the physical arrangement of lineages across species. In anticipation, our current efforts include testing different criteria for defining matches between genome and SolaR80 sequences. A complete genome sequence will also enable detailed in silico analysis of the $R$-gene space of both species. As $R$-gene sequences are discovered, they will be integrated into the SolaR80 framework.

Improved disease resistance remains an important goal for potato and tomato breeders, and wild Solanum species are important sources of resistance genes. In recent years, the utility of cross-species comparative genomics approaches has been demonstrated by the mapping and cloning of several Solanum $R$ genes. With the release of whole-genome sequences of both cultivated potato and tomato, more detailed analyses of the $R$-gene space of both species will ensue. The research presented here provides the research community with a flexible, practical framework for cross-species comparison and analysis of $R$ genes from Solanum species. Through sequence and DNA hybridization experiments, we have demonstrated that most $R$-gene lineages are of ancient origin that predates speciation events. Thus, we predict, comparative genomics approaches to $R$-gene mapping, and cloning will be largely successful across $R$-gene lineages and Solanum species boundaries. Our analyses also demonstrate a need for more thorough sampling of the $R$-gene and Rallele space of Solanum species. Finally, our work provides an illustrative example of how cross-species comparisons can yield hypotheses of the importance of specific $R$-gene lineages in specific Solanum species. Such hypotheses may drive informed phenotypic surveys of Solanum germplasm. 


\section{MATERIALS AND METHODS}

\section{Solanum bulbocastanum RGA.}

Solanum bulbocastanum PI 243510 genotype PT29, provided by J. Helgeson (USDA-ARS, University of Wisconsin, Madison, U.S.A.), was the source of all RGA PCR templates including genomic DNA and cDNA. For genomic DNA, two to three small, young, healthy, fully expanded leaves were harvested from the apex of plants grown under greenhouse conditions (St. Paul, MN, U.S.A.), and DNA extraction was performed using the procedure described by Fulton and associates (1995). For cDNA preparations, two to three small, young, healthy, fully expanded leaves were harvested from the apex of $S$. bulbocastanum plants grown under greenhouse conditions, were immediately flash frozen in liquid nitrogen, and were stored at $-80^{\circ} \mathrm{C}$. Total RNA was extracted using the Wizard SV total RNA isolation kit (Promega Corp., Madison, WI, U.S.A.) following manufacturer's recommendations. First-strand cDNA synthesis was performed, using SuperScriptII reverse transcriptase (Invitrogen, Carlsbad, CA, U.S.A.) and an oligo-dT primer (Integrated DNA Technologies, Coralville, IA, U.S.A.) following manufacturer's recommendations.

RGA primer pairs are listed in Table 1 and include three pairs (SA, SM, and PP) that have been described previously (Leister et al. 1996; Lopez et al. 2003). Two additional primers, bliC1 and bliF1 (primer pair bb; Table 1), were newly designed using the consensus-degenerate hybrid oligonucleotide primers (CODEHOP) program (Rose et al. 1998), targeting the P-loop (bliC1) and kinase 2 (bliF1) motifs and flanking sequences. Deduced protein alignments from NBS-LRR $R$ genes that were previously cloned from solanaceous species were used for primer design. These genes included $\mathrm{Mi}-1$ (GenBank accession number AF091048), Hero (AJ457052), and I2 (AF118127) from tomato, $R B$ (Q7XBQ9) from S. bulbocastanum, and Grol (AY196160), Rx (AJ011801), and Gpa2 (AF195939) from potato.

RGA PCR assays were performed in a total volume of $50 \mu \mathrm{l}$ and included $5 \mu \mathrm{l}$ of $10 \times$ PCR buffer (Applied Biosystems, Foster City, CA, U.S.A.), $0.5 \mu$ AmpliTaq DNA polymerase (Applied Biosystems), $5.0 \mu \mathrm{l} 2.5 \mathrm{mM}$ dNTP mixture (Roche Applied Science, Indianapolis, IN, U.S.A.), $1.0 \mu$ l each of 10 $\mathrm{mM}$ primer (Table 1), and $400 \mathrm{ng}$ of template. Thermocycler (GeneAmp PCR System 9700 or 2700; Applied Biosystems) conditions were an initial denaturation step of $2 \mathrm{~min}$. at $94^{\circ} \mathrm{C}$, followed by 35 cycles of $45 \mathrm{~s}$ at $94^{\circ} \mathrm{C}, 45 \mathrm{~s}$ at $45^{\circ} \mathrm{C}$, and $1 \mathrm{~min}$ $20 \mathrm{~s}$ at $72^{\circ} \mathrm{C}$, and a final extension of $10 \mathrm{~min}$ at $72^{\circ} \mathrm{C}$. PCR products were electrophoresed on a $2 \%$ horizontal Tris-acetateEDTA agarose gel and were stained with ethidium bromide (EtBr) for visualization under UV light.

Amplicons of approximately 500 to $800 \mathrm{bp}$, generated using primer pairs SA, SM, and PP, and amplicons of approximately 300 to $800 \mathrm{bp}$, generated using primer pair bb, were excised from gels, using clean glass microscope slide cover slips. Fragment DNA was extracted from the agarose gel, using the QIAquick gel extraction kit (Qiagen Inc., Valencia, CA, U.S.A.) following manufacturer's instructions, and was cloned into the pGEM-T EZ vector cloning kit (Promega Corp.). White colonies for each primer pair by template combination were manually picked, using sterile micropippette tips, into 3 $\mathrm{ml}$ of liquid Luria-Bertani (LB) media with ampicillin (50 $\mathrm{mg} / \mathrm{ml}$ ), and were incubated overnight at $37^{\circ} \mathrm{C}$, with shaking. Plasmid DNA was extracted from $2 \mathrm{ml}$ of overnight culture, using the Wizard SVS miniprep kit (Promega Corp.). Plasmid DNA was digested to confirm the presence of an insert by combining $4 \mu \mathrm{l}$ of DNA, $2 \mu \mathrm{l}$ of sterile water, $1 \mu \mathrm{l}$ of EcoRI (Invitrogen), and $1 \mu \mathrm{l}$ of digestion buffer and incubating for 1 $\mathrm{h}$ at $37^{\circ} \mathrm{C}$. The entire digestion reaction was electrophoresed on a $1 \%$ horizontal tris-borate-EDTA agarose gel, was stained with $\mathrm{EtBr}$, and was visualized over UV light. Each approximately 300 to $800-$ bp insert (depending on primer pair used) was sequenced in both directions, using M13 forward and reverse sequencing primers (Integrated DNA Technologies) by the University of Minnesota BioMedical Genomics Center (St. Paul, MN, U.S.A.). All S. bulbocastanum RGA sequences have been deposited in GenBank.

\section{DNA and protein sequence analyses.}

Vector sequences were trimmed from individual S. bulbocastanum RGA clone sequences using the SeqMan Pro module of the Lasergene software package (DNAStar, Madison, WI, U.S.A.). DNA sequences were translated using the Translate Tool on the Expert Protein Analysis System (ExPASy) Proteomics Server of the Swiss Institute of Bioinformatics. DNA sequences were subjected to BLASTn and tBLASTx searches, and protein sequences were subjected to BLASTp searches. Requirements for classification of sequences as RGA included a best BLAST match to an $R$ gene or RGA sequence with a value $\leq 1 \mathrm{e}^{-10}$ and the presence of encoded internal amino acid motifs characteristic of $R$ genes and RGA. Classification of sequences into other groups was based on best BLAST hits regardless of $e$-values.

RGA recovered from $S$. tuberosum (Leister et al. 1996), $S$. lycopersicum (Pan et al. 2000), S. habrochaites (Nino-Liu et al. 2003), S. aculeatissimum (syn. S. khasianum [Zhuang and Wang 2009]), S. caripense (Trognitz and Trognitz 2005), S. demissum (J. Zhou, Y. Zhang, X. Pei, Y. Song, Z. Wang, and S. Jia, unpublished), S. melongena (T. Kiran Babu, K. Raghuprakash, S. Sivaramakrishnan, K. Varaprasad, G. Anuradha, and P. Jayamma, unpublished), and S. phureja $\times S$. stenotomum (I. Simko and R. W. Jones, unpublished) were also used for sequence and phylogenetic analyses. Note that the taxonomic status of $S$. phureja and S. stenotomum is questionable and these taxa may, in fact, represent selections from within S. tuberosum (Huaman and Spooner 2002; Spooner et al. 2005). In this study, we retain the " $S$. phureja $\times S$. stenotomum" terminology employed in GenBank. For consistency across studies, to be defined as RGA in the current study, all sequences were held to the criteria employed for S. bulbocastanum; sequences not fitting these criteria were removed from further study. A total of 472 cloned solanaceous NBS-LRR $R$ genes and $R$-gene homologs were also analyzed in this study. For each, NBS sequence was manually extracted for downstream analyses on the basis of comparisons to RGA sequences. A comprehensive list of sequences used in this study is presented in Supplementary Table S1.

SeqMan Pro was used to assemble DNA sequences into contigs based on $80 \%$ sequence identity. The "Classic Assembler" was employed with default assembly parameters. We assembled $80 \%$ contigs for $S$. bulbocastanum RGA alone (Sblb80 diversity bins) and for all 788 RGA, $R$ gene, and $R$ gene homolog sequences together (SolaR80 diversity bins). Individual DNA sequences and SolaR80 consensus sequences were translated using the ExPASy online translation tool and were subjected to BLASTp searches to confirm continued homology and the presence of conserved NBS motifs. Sblb80 and Sola$\mathrm{R} 80$ diversity bins were defined based on analyses conducted on July 18, 2011.

To generate phylogenetic trees, translations were trimmed to the P-loop motif on the $5^{\prime}$ end and the GLPL motif on the $3^{\prime}$ end. Trimmed sequences were aligned using MUSCLE version 3.8 (Edgar 2004). Translated sequence of human Apoptotic protease activating factor 1 (Apaf1) (GenBank accession number AJ243005.1, nucleotides 466 to 972) was included as an outgroup. Alignments were manually edited by anchoring align- 
ments at conserved NBS sequence motifs and domains (P-loop, RNBS-A, Kinase 2, RNBS-B, RNBS-C, and GLPL) and by adjusting flanking sequences as necessary. Complete SolaR80 protein alignments are available as Supplementary Table S2. Alignments were used to create neighbor-joining and fast heuristic parsimony trees using PAUP* 4.0 Macintosh Beta 10 (Sinauer Associates, Inc. Publishers, Sunderland, MA, U.S.A.). Bootstrap values were calculated using default settings and 1,000 bootstrap replications.

$\mathrm{Ka} / \mathrm{Ks}$ ratios were calculated using the Bergen Center for Computational Science online calculator and default settings.

To identify candidate NBS-containing disease resistance genes from the published genome sequence of $S$. tuberosum (Potato Genome Sequencing Consortium 2011), we used a series of string searches to survey reported gene annotations. Search terms were "CC-NB-LRR," "CC-NBS-LRR," "Coiledcoil domain," "Coiled-coil-helix," "Disease resistance," "NBARC," "NBS domain," "NBS-coding," "NBS-LRR," "Nucleotide binding," "Nucleotide binding site-leucine rich repeat," "TIR," "TIR-NBS," and "TIR-NBS-LRR." Identified genes were subjected to translation, motif searches, and assemblage into DNA sequence homology bins as described above.

\section{DNA cross-hybridization experiments.}

Solanum bulbocastanum RGA clones were manually arrayed onto nylon membrane (Hybond N+; GE Healthcare Life Sciences, Piscataway, NJ, U.S.A.) and were grown overnight on $\mathrm{LB}$ medium at $37^{\circ} \mathrm{C}$. Membrane processing, probe labeling, and hybridization methods have been previously described (Bradeen et al. 2003). Blots containing DNA from arrayed BAC libraries were obtained from the Arizona Genomics Institute (Tucson, AZ, U.S.A.) (S. bulbocastanum genotype PT29, S. lycopersicum 'Heinz 1706', and S. melongena 'Black Beauty') or were a gift from B. Baker (USDA-ARS and the University of California [Berkeley, CA, U.S.A.], S. demissum). Hybridizations to BAC blots have been previously described (Bradeen et al. 2003). Probes consisted of pools of equal molar amounts of insert DNA from $S$. bulbocastanum RGA clones.

DNA for Southern genomic analyses was isolated from $S$. melongena 'Black Beauty', S. melongena USDA ARS-GRIN accession PI180485 (an Asian landrace of S. melongena), a putative wild eggplant relative, S. undatum (Meyer \#325), and a wild species distantly related to cultivated eggplant, $S$. violaceum (Meyer \#308). DNA of $S$. bulbocastanum line PT29 was used as a positive control. For each lane, 20 to $25 \mu \mathrm{g}$ of DNA was singly digested for $6 \mathrm{~h}$ with $120 \mathrm{U}$ of restriction enzyme (EcoRI, BamHI, and KpnI [Roche Applied Science]) with appropriate buffer in a $30-\mu l$ reaction. Digested genomic DNA was separated on a $0.9 \%$ agarose gel and was transferred to positively charged nylon membrane (Roche Applied Science) by capillary blotting (Sambrook and Russell 2001). Probes were composed of pools of equal molar amounts of insert DNA from $S$. bulbocastanum RGA clones and were labeled with digoxigenin, using the PCR DIG probe synthesis kit (Roche Applied Science) following manufacturer's protocols. A $S$. bulbocastanum BAC blot known to hybridize with the probes was included as a positive control. Probe were hybridized at $42^{\circ} \mathrm{C}$, using reagents from Roche Applied Science according to manufacturer's protocols. Bands were visualized in a solution of nitro blue tetrazolium chloride and 5-bromo-4chloro-3-indolyl phosphate, and blots were photographed using a Canon Rebel XS camera. Membranes were stripped and were rehybridized, following the DIG Easy Hyb granules protocol (Roche Applied Science) with one modification, i.e., stripping was preceded by incubation in a room temperature bath of $0.4 \mathrm{M} \mathrm{NaOH}$ with $0.1 \%$ sodium dodecyl sulfate for 5 $\min$.

\section{ACKNOWLEDGMENTS}

This work was supported by the Plant Genome Program of the United States Department of Agriculture National Institute of Food and Agriculture (USDA NIFA). Computer and software support by the University of Minnesota Supercomputing Institute is gratefully acknowledged. The authors thank J. Helgeson and B. Baker for sharing plant materials and BAC library filters. This is contribution \#257 from the Department of Soil, Plant, Environmental and Animal Production Sciences, University of Naples Federico II.

\section{LITERATURE CITED}

Austin, S., Pohlman, J. D., Brown, C. R., Mojtahedi, H., Santo, G. S. Douches, D. S., and Helgeson, J. P. 1993. Interspecific somatic hybridization between Solanum tuberosum L. and S. bulbocastanum Dun. as a means of transferring nematode resistance. Am. Potato J. 70:485-495.

Bonierbale, M. W., Plaisted, R. L., and Tanksley, S. D. 1988. RFLP maps based on a common set of clones reveal modes of chromosomal evolution in potato and tomato. Genetics 120:1095-1103.

Bradeen, J. M. 2011. Cloning of late blight resistance genes: Strategies and progress. In: Genetics, Genomics and Breeding of Potato, J. M. Bradeen and C. Kole, eds. CRC Press/Science Publishers, Enfield, NH, U.S.A.

Bradeen, J. M., Naess, S. K., Song, J., Haberlach, G. T., Wielgus, S. M., Buell, C. R., Jiang, J., and Helgeson, J. P. 2003. Concomitant reiterative $\mathrm{BAC}$ walking and fine genetic mapping enable physical map development for the broad-spectrum late blight resistance region, $R B$. Mol. Gen. Genet. 269:603-611.

Brown, C. R., Yang, C. P., Mojtahedi, H., Santo, G. S., and Masuelli, R. 1996. RFLP analysis of resistance to Columbia root-knot nematode derived from Solanum bulbocastanum in a BC2 population. Theor. Appl. Genet. 92:572-576.

Brown, C. R., Mojtahedi, H., Zhang, L.-H., and Riga, E. 2009. Independent resistant reactions expressed in root and tuber of potato breeding lines with introgressed resistance to Meloidogyne chitwoodi. Phytopathology 99:1085-1089.

Brugmans, B., Wouters, D. van Os, H., Hutten, R. van der Linden, G., Visser, R. G. F., Van Eck, H. J., and Van der Vossen, E. A. G. 2008. Genetic mapping and transcription analyses of resistance gene loci in potato using NBS profiling. Theor. Appl. Genet. 117:1379-1388.

Cannon, S. B., Zhu, H., Baumgarten, A. M., Spangler, R., May, G., Cook, D. R., and Young, N. D. 2002. Diversity, distribution, and ancient taxonomic relationships within the TIR and non-TIR NBS-LRR resistance gene subfamilies. J. Mol. Evol. 54:548-562.

Couch, B. C., Spangler, R., Ramos, C., and May, G. 2006. Pervasive purifying selection characterizes the evolution of $I 2$ homologs. Mol. PlantMicrobe Interact. 19:288-303.

Doganlar, S., Frary, A., Daunay, M.-C., Lester, R. N., and Tanksley, S. D. 2002. A comparative genetic linkage map of eggplant (Solanum melongena) and its implications for genome evolution in the Solanaceae. Genetics 161:1697-1711.

Edgar, R. C. 2004. MUSCLE: A multiple sequence alignment method with reduced time and space complexity. BMC Bioinformatics 5:113.

Ellis, J. G., Lawrence, G. J., Luck, J. E., and Dodds, P. N. 1999. Identification of regions in alleles of the flax rust resistance gene $L$ that determine differences in gene-for-gene specificity. Plant Cell 11:495-506.

Fulton, T. M., Chunwongse, J., and Tanksley, S. D. 1995. Microprep protocol for extraction of DNA from tomato and other herbaceous plants. Plant Mol. Biol. Rep. 13:207-209.

Gebhardt, C., and Valkonen, J. P. T. 2001. Organization of genes controlling disease resistance in the potato genome. Ann. Rev. Phytopath. 39:79-102.

Grube, R. C., Radwanski, E. R. and Jahn, M. 2000. Comparative genetics of disease resistance within the Solanaceae. Genetics 155:873-887.

Hattendorf, A., and Debener, T. 2007. Molecular characterization of NBSLRR-RGA in the rose genome. Physiol. Plant 129:775-786.

He, L., Du, C., Covaleda, L., Xu, Z., Robinson, A. F., Yu, J. Z., Kohel, R. J., and Zhang, H. 2004. Cloning, characterization, and evolution of the NBS-LRR-encoding resistance gene analogue family in polyploid cotton (Gossypium hirsutum L.). Mol. Plant-Microbe Interact. 17:1234-1241.

Huaman, Z., and Spooner, D. M. 2002. Reclassification of landrace populations of cultivated potatoes (Solanum sect. Petota). Am. J. Bot. 89:947-965.

Leister, D., Ballvora, A., Salamini, F., and Gebhardt, C. 1996. A PCRbased approach for isolating pathogen resistance genes from potato with potential for wide application in plants. Nat. Genet. 14:421-429.

Liberles, D. A. 2001. Evaluation of methods for determination of a reconstructed history of gene sequence evolution. Mol. Biol. Evol. 18:20402047. 
Livingstone, K. D., Lackney, V. K., Blauth, J. R., van Wijk, R., and Jahn, M. K. 1999. Genome mapping in Capsicum and the evolution of genome structure in the Solanaceae. Genetics 152:1183-1202.

Lokossou, A. A., Park, T., van Arkel, G., Arens, M., Ruyter-Spira, C., Morales, J., Whisson, S. C., Birch, P. R. J., Visser, R. G. F., Jacobsen, E., and van der Vossen, E. A. G. 2009. Exploiting knowledge of R/Avr genes to rapidly clone a new LZ-NBS-LRR family of late blight resistance genes from potato linkage group IV. Mol. Plant-Microbe Interact. 22:630-641.

Lopez, C. E., Acosta, I. F., Jara, C., Pedraza, F., Gaitan-Solis, E., Gallego, G., Beebe, S., and Tohme. J. 2003. Identifying resistance gene analogs associated with resistances to different pathogens in common bean. Phytopathology 93:88-95.

Maleki, L., Faris, J. D., Bowden, R. L., Gill, B. S., and Fellers, J. P. 2003. Physical and genetic mapping of wheat kinase analogs and NBS-LRR resistance gene analogs. Crop Sci. 43:660-670.

Mazourek, M., Cirulli, E., Collier, S. M., Landry, L. G., Kang, B.-C., Quirin, E. A., Bradeen, J. M., Moffett, P. and Jahn, M. 2009. The fractionated orthology of $B s 2$ and $R x / G p a 2$ and the comparative genomics model of disease resistance in the Solanaceae. Genetics 182:1351-1364.

Meyers, B. C., Dickerman, A. W., Michelmore, R. W., Sivaramakrishnan, S., Sobral, B. W., and Young, N. D. 2002. Plant disease resistance genes encode members of an ancient and diverse protein family within the nucleotide-binding superfamily. Plant J. 20:317-332.

Meyers, B. C., Kozik, A., Griego, A., Kuang, H., and Michelmore, R. W. 2003. Genome-wide analysis of NBS-LRR-encoding genes in Arabidopsis. Plant Cell 15:809-934.

Michelmore, R. W., and Meyers, B. C. 1998. Clusters of resistance genes in plants evolve by divergent selection and a birth-and-death process. Genome Res. 8:1113-1130.

Moffett, P., Farnham, G., Peart, J., and Baulcombe, D. C. 2002. Interaction between domains of a plant NBS-LRR protein in disease resistancerelated cell death. EMBO (Eur. Mol. Biol. Organ.) J. 21:4511-4519.

Nino-Liu, D.O., Zhang, L., and Foolad, M. R. 2003. Sequence comparison and characterization of DNA fragments amplified by resistance gene primers in tomato. Acta Hortic 625:49-58.

Oosumi, T., Rockhold, D. R., Maccree, M. M., Deahl, K. L., McCue, K F., and Belknap, W. R. 2009. Gene Rpi-bt1 from Solanum bulbocastanum confers resistance to late blight in transgenic potatoes. Am. J. Pot. Res. 86:456-465.

Pan, Q., Liu,Y. S., Budai Hadrian, O., Sela, M., Carmel Goren, L., Zamir, D., and Fluhr, R. 2000. Comparative genetics of nucleotide binding site-leucine rich repeat resistance gene homologues in the genomes of two dicotyledons: Tomato and Arabidopsis. Genetics 155:309-322.

Potato Genome Sequencing Consortium. 2011. Genome sequence and analysis of the tuber crop potato. Nature 475:189-195.

Prince, J. P., Pochard, E., and Tanksley, S. D. 1993. Construction of a molecular linkage map of pepper and a comparison of synteny with tomato. Genome 36:404-417.

Rose, T. M., Schultz, E. R., Henikoff, J. G., Pietrokovski, S., McCallum, C. M., and Henikoff, S. 1998. Consensus-degenerate hybrid oligonucleotide primers for amplificaiton of distantly-related sequences. Nucleic Acids Res. 26:1628-1635.

Sambrook, J., and Russell, D. W. 2001. Molecular Cloning: A Laboratory Manual. Third ed. Cold Spring Harbor Laboratory Press, Cold Spring Harbor, NY, U.S.A.

Siltberg, J., and Liberles, D. A. 2002. A simple covarion-based approach to analyse nucleotide substitution rates. J. Evol. Biol. 15:588-594.

Song, J., Bradeen, J. M., Naess, S. K., Raasch, J. A., Wielgus, S. M., Haberlach, G. T., Liu, J., Kuang, H., Austin-Phillips, S., Buell, C. R.,
Helgeson, J. P., and Jiang, J. 2003. Gene $R B$ from Solanum bulbocastanum confers broad spectrum resistance against potato late blight pathogen Phytophthora infestans. Proc. Natl. Acad. Sci. U.S.A. 100:9128-9133.

Spooner, D. M., Anderson, G. J. and Jansen, R. K. 1993. Chloroplast DNA evidence for the interrelationships of tomatoes, potatoes, and pepinos (Solanaceae). Am. J. Bot. 80:676-688.

Spooner, D. M., McLean, K., Ramsay, G., Waugh, R., and Bryan, G. J. 2005. A single domestication for potato based on multilocus amplified fragment length polymorphism genotyping. Proc. Natl. Acad. Sci. U.S.A. 102:14694-14699.

Stevenson, W. R., Loria, R., Franc, G. D., and Weingartner, D. P. 2001. Compendium of Potato Diseases. 2nd ed. American Phytopathological Society Press, St. Paul, MN, U.S.A.

Stupar, R.M., Song, J., Tek, A. L., Cheng, Z., Dong, F., and Jiang, J. 2002. Highly condensed potato pericentromeric heterochromatin contains rDNA-related tandem repeats. Genetics 162:1435-1444.

Tameling, W. I. L., Elzinga, S. D. J., Darmin, P. S., Vossen, J. H., Takken, F. L. W., Haring, M. A., and Cornelissen, B. J. C. 2002. The tomato $R$ gene products I-2 and Mi-1 are functional ATP binding proteins with ATPase activity. Plant Cell 14:2929-2939.

Trognitz, F., and Trognitz, B. 2005. Survey of resistance gene analogs in Solanum caripense, a relative of potato and tomato, and update on $R$ gene genealogy. Mol. Genet. Genomics 274:595-605.

van der Vossen, E., Sikkema, A., Hekkert, B. T. L. Gros, J., Stevens, P., Muskens, M., Wouters, D., Pereira, A., Stiekema, W., and Allefs, S. 2003. An ancient R gene from the wild potato species Solanum bulbocastanum confers broad-spectrum resistance to Phytophthora infestans in cultivated potato and tomato. Plant J. 37:867-882.

van der Vossen, E. A. G., Gros, J., Sikkema, A., Muskens, M., Wouters, D., Wolters, P., Pereira, A., and Allefs, S. 2005. The Rpi-blb2 gene from Solanum bulbocastanum is an $\mathrm{Mi}-1$ gene homolog conferring broadspectrum late blight resistance in potato. Plant J. 44:208-222.

Wang, M., Van den Berg, R., Van der Linden, G., and Vosman, B. 2008. The utility of NBS profiling for plant systematics: A first study in tuberbearing Solanum species. Plant Syst. Evol. 276:137-148.

Weese, T. L., and Bohs, L. 2007. A three-gene phylogeny of the genus Solanum (Solanaceae). Syst. Bot. 32:445-463.

Whitaker, V. M., Bradeen, J. M., Debener, T., Biber, A., and Hokanson, S. C. 2010. Rdr3, a novel locus conferring black spot disease resistance in tetraploid rose: Genetic analysis, LRR profiling, and SCAR marker development. Theor. Appl. Genet. 120:573-585.

Wikstrom, N., Savolainen, V., and Chase, M. W. 2001. Evolution of the angiosperms: Calibrating the family tree. Proc. Biol. Sci. 268:22112220

Zhang, L. P., Khan, A., Nino-Liu, D., and Foolad, M. R. 2002. A molecular linkage map of tomato displaying chromosomal locations of resistance gene analogs based on a Lycopersicon esculentum $\times$ Lycopersicon hirsutum cross. Genome 45:133-146.

Zhou, T., Wang, Y., Chen, J. Q., Araki, H., Jing, Z., Jiang, K., Shen, J., and Tian, D. 2004. Genome-wide distribution of NBS genes in japonica rice reveals significant expansion of divergent non-TIR NBS-LRR genes. Mol. Gen. Genomics 271:402-415.

Zhuang, Y., and Wang, S. 2009. Isolation and analysis of resistance gene analogs from Solanum khasianum. Mol. Plant Breed. 7:1223-1228.

\section{AUTHOR-RECOMMENDED INTERNET RESOURCE}

USDA-ARS National Plant Germplasm System website: www.ars-grin.gov/npgs/index.html 